\title{
Assessment of the use of ED Chief Complaint Data for monitoring Chronic Diseases
}

\author{
Megan T. Patel* \\ Epidemiology and Biostatistics, University of Illinois Chicago, Chicago, IL, USA
}

\section{Objective}

To create chronic disease categories for emergency department (ED) chief complaint data and evaluate the categories for validity against ED data with discharge diagnoses and hospital discharge data.

\section{Introduction}

Syndromic Surveillance (SS), traditionally applied to infectious diseases, is more recently being adapted to chronic disease prevention. Its usefulness rests on the large number of diverse individuals visiting emergency rooms with the possibility of real-time monitoring of acute health effects, including effects from environmental events and its potential ability to examine more long-term health effects and trends of chronic diseases on a local level [1-3].

\section{Methods}

Emergency department chief complaint (CC) data captured by the Cook County Department of Public Health local instance of ESSENCE from Jan 1, 2006 - Dec 31, 2013 was utilized to generate chronic disease categories for: CVD, AMI, ACS, angina, stroke, diabetes, hypertension, asthma, and COPD based on disease symptoms, natural language processing for free text chief complaints, and associated terms present in EMR system menus.

A standard category was created for each chronic disease category based on discharge diagnoses (ICD-9 code), and their associated terms. The ICD-9 based categories were applied to the discharge diagnosis field within the ED data. The chief complaint based chronic disease category definitions were compared to the standard classification by determining the sensitivity, specificity, positive predictive value, and negative predictive value.

The standard chronic disease categories created with ICD-9 codes for the chronic disease category validation were also applied to Illinois hospital discharge data for Cook County from Jan 1, 2006 - Dec 31, 2013. This data was compared to the chief complaint categories from the ED data for the same time period by visual analysis through time series and strength of correlation by Pearson correlation coefficient analysis. ESSENCE version 1.17 was utilized for the free-text query development and SAS 9.4 was utilized to perform the analyses.

\section{Results}

For the validation analysis, 1,366,525 (24.76\%) ED visits of individuals 40 years and older and 867,509 (15.72\%) ED visits of individuals less than 18 years of age with a valid chief complaint and discharge diagnosis were included. Validation results are presented in Table 1. Specificity was generally high for most of the categories, with the narrow definitions having a higher specificity (Narrow AMI $=0.9996$, Broad AMI $=0.9119$ ). However, the loss in sensitivity is substantial in moving from the broader definition to the narrow definition $($ Broad AMI $=0.5444$, Narrow AMI $=0.1040)$. The positive predictive values had a wide range from 0.0128 for the Broad ACS category to 0.7199 for the Narrow Asthma definition. The negative predictive values were high for all chronic disease categories ranging from 0.9501 for the Narrow CVD category to 0.9996 for Angina.

The Pearson correlation coefficients are presented in Table 2. Graphs showing the comparisons of the chief complaint based ED data to the hospitalization data by chronic disease category definition are presented in Figure 1. Pearson correlations ranged from 0.9323 for Narrow Asthma to 0.1992 for Hypertension.

\section{Conclusions}

Based on the high specificity and correlation coefficients in comparison to hospital discharge data, emergency department chief complaint data captured with syndromic surveillance could be utilized to examine chronic disease categories: asthma, COPD, CVD, AMI, ACS, stroke, and diabetes at a local, state or national level.

\begin{tabular}{|c|c|c|c|c|c|}
\hline Chronic Disease Category & Sensitivity & Specificity & PPV & NPV & Prevalence (\%) \\
\hline Broad CVD & 0.4686 & 0.9112 & 0.3154 & 0.9515 & 11.93 \\
\hline Narrow CVD & 0.4455 & 0.9213 & 0.3308 & 0.9501 & 10.81 \\
\hline Narrow AMI & 0.1040 & 0.9996 & 0.4362 & 0.9973 & 0.07 \\
\hline Broad AMI & 0.5444 & 0.9119 & 0.0186 & 0.9837 & 8.95 \\
\hline Narrow ACS & 0.0939 & 0.9981 & 0.0634 & 0.9988 & 0.20 \\
\hline Broad ACS & 0.7256 & 0.9248 & 0.0128 & 0.9996 & 7.60 \\
\hline Angina & 0.7257 & 0.9255 & 0.0133 & 0.9996 & 7.54 \\
\hline Broad Stroke 1 & 0.3034 & 0.9820 & 0.2186 & 0.9884 & 2.27 \\
\hline Broad Stroke 2 & 0.2439 & 0.9910 & 0.3116 & 0.9875 & 1.28 \\
\hline Narrow Stroke & 0.1623 & 0.9983 & 0.6089 & 0.9863 & 0.44 \\
\hline Hypertension & 0.3068 & 0.9914 & 0.4803 & 0.9822 & 1.61 \\
\hline Broad Diabetes & 0.1388 & 0.9930 & 0.1595 & 0.9918 & 0.82 \\
\hline Narrow Diabetes & 0.0458 & 0.9976 & 0.1523 & 0.9910 & 0.28 \\
\hline Broad COPD & 0.6596 & 0.9456 & 0.1188 & 0.9960 & 6.11 \\
\hline Narrow COPD & 0.1092 & 0.9992 & 0.6104 & 0.9902 & 0.20 \\
\hline Narrow Asthma & 0.3855 & 0.9959 & 0.7119 & 0.9839 & 1.40 \\
\hline Broad Asthma & 0.4751 & 0.9861 & 0.5750 & 0.9861 & 2.14 \\
\hline
\end{tabular}

Table 2. Pearson correlation coefficients for the comparison of ED chief complaint based chronic disease

categories with hospitalization data by chronic disease category.
Chronic Disease Category Pearson Correlation

\begin{tabular}{cc}
\hline Chronic Disease Category & $\begin{array}{c}\text { Pearson Correlation } \\
(\mathbf{p} \text { value) }\end{array}$ \\
\hline Broad CVD & 0.4950 \\
& $(<0.0001)$ \\
Narrow CVD & 0.4902 \\
& $(<0.0001)$ \\
Narrow AMI & 0.2666 \\
& $(0.0086)$ \\
Broad AMI & 0.4278 \\
& $(<0.0001)$ \\
Narrow ACS & 0.3169 \\
& $(0.0017)$ \\
Broad ACS & 0.3542 \\
& $(0.0004)$ \\
Angina & 0.4305 \\
& $(<0.0001)$ \\
Broad Stroke 1 & 0.2556 \\
& $(0.012)$ \\
Broad Stroke 2 & 0.5655 \\
& $(<0.0001)$ \\
Narrow Stroke & 0.3525 \\
& $(0.0004)$ \\
Hypertension & 0.0573 \\
& $(0.5796)$ \\
Broad Diabetes & 0.4781 \\
& $(<0.0001)$ \\
Narrow Diabetes & 0.5351 \\
& $(<0.0001)$ \\
Broad COPD & 0.5549 \\
& $(<0.0001)$ \\
Narrow COPD & 0.6513 \\
& $(<0.0001)$ \\
Narrow Asthma & 0.9302 \\
& $(<0.0001)$ \\
Broad Asthma & 0.9158 \\
& $(<0.0001)$ \\
\hline & \\
&
\end{tabular}




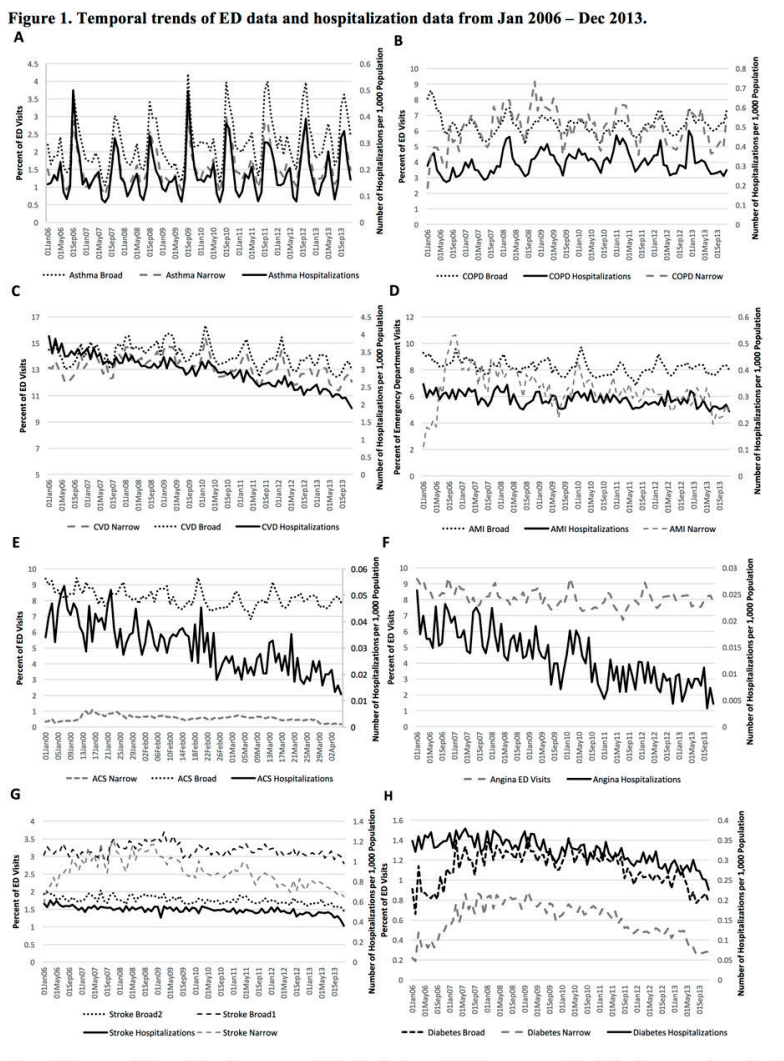

Figure 1: Emergency visits are depicted as percentage of ED visits for the specificd discasc catcgory. Hospitalization rates are the number of hospitalizations per 1,000 population. Asthma ED visits and hospitalizations were only for individuals less than 18 years of age. All other disea
categories were adults aged greater than 39 years. A) Asthma, B) COPD, C) CVD D) AMI, E) ACS, F) Angina, G) Stroke, and H) Diabetes.

\section{Keywords}

chronic disease; evaluation; ED data; syndromic surveillance

\section{Acknowledgments}

Demian Christiansen, Kelley Bemis, and Victoria Persky

\section{References}

1. Bassil, K.L., et al., Temporal and spatial variation of heat-related illness using 911 medical dispatch data. Environ Res, 2009. 109(5): p. 600-6.

2. Mathes, R.W., K. Ito, and T. Matte, Assessing syndromic surveillance of cardiovascular outcomes from emergency department chief complaint data in New York City. PLoS One, 2011. 6(2): p. e14677.

3. Zanobetti, A. and J. Schwartz, Air pollution and emergency admissions in Boston, MA. J Epidemiol Community Health, 2006. 60(10): p. 890-5.

\section{*Megan T. Patel}

E-mail:mtoth2@uic.edu 